\title{
The Biosynthesis of Spinosyn in Saccharopolyspora spinosa: Synthesis of the Cross-Bridging Precursor and Identification of the Function of SpnJ
}

\author{
Hak Joong Kim, Rongson Pongdee, Qingquan Wu, Lin Hong, and Hung-wen Liu \\ Division of Medicinal Chemistry, College of Pharmacy, and Department of Chemistry and \\ Biochemistry, University of Texas at Austin, Austin, Texas 78712, E-mail: h.w.liu@mail.utexas.edu
}

\begin{abstract}
Spinosyns are glycosylated polyketide-derived macrolides possessing a perhydro- $a$ s-indacene core that is presumably formed via a series of intramolecular cross-bridging reactions. The unusual structure of the spinosyn aglycone suggests an intriguing biosynthetic pathway for its formation, which is expected to be initiated by the oxidation of the $15-\mathrm{OH}$ group of the mature polyketide precursor and may involve a "Diels-Alder" type [4+2] cycloaddition reaction. Three possible routes, which differ in the order of oxidation and cyclization events, can be envisioned for the biosynthesis of the core structure. Sequence analysis of the spinosyn biosynthetic gene cluster led to the speculation of spnJ as the possible oxidase gene. To explore the early stage of intramolecular ring formation, we cloned and expressed the spnJ gene and purified the SpnJ protein which shows the characteristics of flavoproteins. Two possible substrates for SpnJ, the linear mature polyketide precursor and the corresponding cyclized macrolactone, were also synthesized. TLC and HPLC analysis of the incubation mixture of these compounds with SpnJ revealed that only the synthesized macrolactone could be converted to the corresponding ketone. This result clearly indicated that macrolactone formation proceeds $15-\mathrm{OH}$ oxidation since the linear polyketide is not a substrate for SpnJ. In summary, the experiments described herein detail a convergent synthesis of spinosyn macrolactone and validate the catalytic function of SpnJ as a flavin-dependent oxidase. More significantly, we have established the spinosyn macrolactone as the immediate precursor of the tricyclic nucleus of spinosyns.
\end{abstract}

Spinosyns A (1) and D (2) are polyketide-derived macrolides produced by Saccharopolyspora spinosa. ${ }^{1}$ The combination of $\mathbf{1}$ and $\mathbf{2}$ serves as the active ingredient in a commercial insecticide, Naturalyte. This mixture exhibits excellent insecticidal activity with low mammalian toxicity and little detrimental environmental effects. ${ }^{2}$ Structurally, the Spinosyns consist of a 22-membered macrolactone ring fused to a perhydro-as-indacene core scaffold. In addition, they are glycosylated with tri- $O$-methylrhamnose and forosamine at $\mathrm{C}-9$ and $\mathrm{C}-17$, respectively. The aglycone portion of Spinosyns is unusual among polyketide-derived secondary metabolites due to the presence of three intramolecular carbon-carbon bonds that constitute the as-indacene skeleton. The unusual nature of the Spinosyn aglycone suggests an intriguing biosynthetic pathway which has generated much recent attention. ${ }^{3,4}$

The Spinosyn (spn) biosynthetic gene cluster was cloned from S. spinosa. ${ }^{3}$ Sequence analysis coupled with gene disruption experiments suggested that macrolactone 4 , which is derived from the linear polyketide precursor 3 , is a likely intermediate to form the $a$-indacene aglycone (9), 3,4 and the proteins encoded by $s p n F$, spnJ, spnL, spnM could be involved in the cross- 
bridging modifications. SpnJ exhibits sequence homology to a flavin dependent dehydrogenase (55\% identity, 68\% similarity) from Streptomyces steffisburgensis, 5 and the SpnF, SpnL and SpnM proteins show resemblance to methyltransferase (SpnF and $\mathrm{SpnL})$ and lipase $(\mathrm{SpnM})$, 3 but lack part of the feature residues/motifs conserved among members of each enzyme family. Hence, their biological functions are not apparent and their roles in the cross-bridging reactions cannot be established with certainty.

If $\mathbf{4}$ is indeed a precursor of the polycyclic aglycone and the cross-bridging reactions are all post-cyclization events, oxidation of the 15-hydroxyl group of $\mathbf{4}$ to a keto group $(\mathbf{4} \rightarrow \mathbf{5})$, and deprotonation at $\mathrm{C}-14$ to form the $\mathrm{C}-3 / \mathrm{C}-14$ linkage $(\mathbf{5} \rightarrow \mathbf{6} \rightarrow \mathbf{7})$ likely occur first (Scheme 1 , route A). Subsequent deprotonation at C-14 of 7 and elimination of the 11-hydroxyl group to give $\mathbf{8}$ is expected to trigger the second cross-coupling reaction $(\mathbf{8} \rightarrow \mathbf{9})$ to produce the asindacene core. However, it is also possible that the initial oxidation of the 15-hydroxyl group takes place on the linear precursor $\mathbf{3}$ when it is still linked to the acyl carrier protein (ACP) associated with the polyketide synthase SpnE (Scheme 1, route B). The keto product 10 is next cleaved from ACP catalyzed by a thioesterase (TE) to give $\mathbf{5}$, which then undergoes sequential cyclizations in a similar manner as described above. In this pathway, compound $\mathbf{4}$ is bypassed.

Although a [4+2] cyclization reaction has been speculated to be a key step in the biosynthesis of many natural products, few have been investigated at the molecular level. ${ }^{6}$ In the case of the polyketide-derived lovastatin (14, Scheme 2$)$, which contains an octahydronaphthalene core, in vitro study with purified polyketide synthase (PKS) showed that it is capable of converting a linear $N$-acetylcysteamine mimic of the appropriate enzyme-bound polyketide intermediate (12) to the correct isomer of the octahydronaphthalene moiety (13). ${ }^{6 \mathrm{~b}, \mathrm{~d}} \mathrm{By}$ analogy to 14, the cross-bridging reactions in the Spinosyn case could also take place when the polyketide intermediate $\mathbf{1 0}$ is still tethered to ACP. In this scenario, C-15 oxidation should occur on the polyketide intermediate $\mathbf{3}$ to give $\mathbf{1 0}$, which would then undergo cyclization to form the tricyclic polyketide 11 (Scheme 1, route C). In this route, the intermediacies of 4-8 are no longer necessary.

Intrigued by the complexity of the tetracyclic nucleus of the Spinosyns, especially the possible involvement of a "Diels-Alder" type [4+2] cyclization $(\mathbf{7} \rightarrow \mathbf{8}$ or $\mathbf{1 0} \rightarrow \mathbf{1 1})$, we decided to study the proposed cross-bridging reactions by first focusing on the initial oxidation step to distinguish the three possible pathways. Herein, we report the expression and purification of SpnJ, the chemical synthesis of $\mathbf{3}$ and $\mathbf{4}$, and the results showing that $\mathbf{4}$, but not $\mathbf{3}$, is a substrate for SpnJ. Our results clearly demonstrate that SpnJ is the predicted oxidase and is specific for $\mathrm{C}-15$ oxidation of macrolactone 4 . These findings provide compelling evidence establishing the early sequence of events in the cross-bridging reactions.

Outlined in Scheme 3 is our retro-synthetic analysis for the preparation of $\mathbf{3}$ and $\mathbf{4}$, in which two main couplings were envisioned: a Julia-Kocienski olefination of sulfone 15 with aldehyde 16 to establish the $E$-alkene geometry at $\mathrm{C}-12 / \mathrm{C}-13$, and a palladium-mediated Heck reaction to connect the C-5/C-6 carbon-carbon bond with 17. Once the linear polyketide 3 is constructed, a Yamaguchi macrolactonization to form the ester linkage at C-1 will afford 4.7

Our synthesis of the aryl sulfone 15, shown in Scheme 4, began with the Weinreb amide $\mathbf{1 8}$ derived from $\delta$-valerolactone. ${ }^{8}$ Asymmetric addition ${ }^{9}$ of diethyl zinc catalyzed by $(-)-(1 S$, $2 R)-N, N$-dibutylnorephedrine [(-)-DBNE] provided 19, whose enantiomeric purity (92\% ee) and absolute configuration were determined by NMR analysis of the corresponding $(R)$ - and $(S)$-Mosher esters. Subsequent silylation followed by reduction furnished $\mathbf{2 0}$. Aldol reaction ${ }^{10}$ between 20 and Crimmins' oxazolidinethione auxiliary 21 gave the syn-aldol adduct 22. The configuration at C-16/C-17 were determined by application of the Zimmerman-Traxler transition state model as well as analysis of the $J_{16,17}$ values, which are in accord with literature 
precedent. ${ }^{11}$ Compound $\mathbf{2 3}$, derived from $\mathbf{2 2}$ via silylation and reduction, was subjected to Ley oxidation and then Brown's (+)-Ipc ${ }_{2} \mathrm{~B}$ (allyl) reagent ${ }^{12}$ to yield $\mathbf{2 4}$ as a single stereoisomer. The relative configurations at C-15/C-17 were determined by conversion of $\mathbf{2 4}$ to the corresponding 1,3-acetonide followed by application of Rychnovsky's ${ }^{13} \mathrm{C}$ NMR method ${ }^{13}$ providing proof for a 1,3-syn relationship. Silylether protection followed by a two-step Lemieux-Johnson oxidation of the terminal olefin and reduction of the resulting aldehyde gave 26. It was crucial to conduct the oxidation of the terminal alkene under neutral $\mathrm{pH}$ as a nonbuffered reaction medium resulted in unwanted deprotection of the triethylsilyl ether at C-15 which required reprotection. Completion of the requisite sulfone 15 was acheived by conversion of $\mathbf{2 6}$ to aryl sulfide $\mathbf{2 7}$ under Mitsunobu conditions followed by oxidation employing hydrogen peroxide and ammonium molybdate. ${ }^{14}$

Our synthesis of aldehyde $\mathbf{1 6}$ is depicted in Scheme 5. Asymmetric Brown allylation of dialdehyde 28, obtained from D-mannitol, ${ }^{15}$ followed by benzoylation of the newly generated secondary alcohols and removal of the isopropylidene protecting group under acidic hydrolysis afforded diol $\mathbf{2 9}$ in excellent overall yield. Oxidative cleavage of the vicinal diol in $\mathbf{2 9}$ facilitated by lead tetraacetate followed by sodium borohydride reduction of the ensuing aldehyde afforded primary alcohol 30. Next, protection of the alcohol functionality in $\mathbf{3 0}$ as its PMB ether followed by exchange of the benzoate protecting groups for silyl ethers provided $\mathbf{3 1}$. Following an oxidation of the terminal olefin in $\mathbf{3 1}$ using Jin's protocol, ${ }^{16}$ the resulting aldehyde was subjected to Takai olefination ${ }^{17}$ affording the desired $E$-vinyliodide 32 in good overall yield as a single stereoisomer. Oxidative removal of the PMB ether employing DDQ in aqueous dichloromethane and oxidation of the liberated hydroxy group with Dess-Martin Periodinane then provided aldehyde $\mathbf{1 6 .}$

With fragments 15 and $\mathbf{1 6}$ in-hand, we assembled the presumed biosynthetic precursors 3 and 4 (Scheme 6). The use of KHMDS in THF provided the best yield and stereoselectivity in forming the $\mathrm{C}(12)-\mathrm{C}(13)$ olefin by the Julia-Kocienski olefination ${ }^{18}$ protocol. Completion of the triene moiety was accomplished by a Heck reaction involving $\mathbf{3 3}$ with ethyl dienoate $17^{19}$ under ligand-free conditions 20 affording 34 as a mixture of isomers (E/Z $=6 / 1$ at $C(4)$ $\mathrm{C}(5))$ that were separated by flash column chromatography. Chemoselective deprotection of the TES ether utilizing PPTS in ethanol followed by saponification furnished seco-acid $\mathbf{3 5}$. Acid 35 was then subject to Yamaguchi macrolactonization ${ }^{21}$ followed by global deprotection of the silicon-based protecting groups employing HF.Pyr in cold ethanol for four days to give 4 in moderate yields. ${ }^{22}$ The preparation of the linear substrate 3 was achieved in a similar manner. Due to the difficulty of preparing the ACP-bound linear polyketide chain, the intended linear substrate 3 was synthesized as the $N$-acetylcysteamine (NAC) thioester $3^{\prime}$. Since the corresponding $N$-acetylcysteamine (NAC) linked thioesters have been commonly used in the biosynthetic studies as surrogates for ACP-bound acyl esters, $\mathbf{3}^{\prime}$ is expected to be an effective substitute for $\mathbf{3}$. As shown in Scheme 6, saponification of $\mathbf{3 4}$ afforded acid 36, which was then coupled with $N$-acetylcysteamine under Yamaguchi esterification conditions. Final deprotection using HF.Pyr complex led to the desired linear substrate $3^{\prime} .{ }^{22}$

With sufficient quantities of $\mathbf{3}^{\prime}$ and $\mathbf{4}$ now available, we evaluated whether the linear polyketide thioester $\mathbf{3}^{\prime}$ or the macrolactone $\mathbf{4}$ is a substrate for SpnJ, which is predicted to be an oxidase. Since the functions of SpnF, SpnL, and SpnM are not obvious, it would be interesting to examine whether SpnJ is a multi-functional enzyme capable of catalyzing not only oxidation at $\mathrm{C}-15$, but also deprotonation at $\mathrm{C}-14$ to initiate the Michael addition to form a $\mathrm{C}-\mathrm{C}$ bond between $\mathrm{C}-3$ and $\mathrm{C}-14$, or the 1,4-elimination of the 11-OH group setting the stage for a possible "Diels-Alder" type [4+2] cycloaddition.

To characterize SpnJ, the $s p n J$ gene was cloned into a pET28b(+) vector and the resulting construct was expressed in E. coli BL21(DE3) cells. ${ }^{22}$ After induction under $25 \mathrm{mM}$ isopropyl 
$\beta$-D-thiogalactopyranoside (IPTG), the $N$-terminal His $_{6}$-tagged $\mathrm{SpnJ}$ protein $(60.6 \mathrm{kDa})$ was purified to near homogeneity using Ni-NTA resin. ${ }^{22}$ The absorption spectrum of the purified protein is typical for that of a flavoprotein. To determine the function of SpnJ, SpnJ (20 $\mu \mathrm{M})$ was incubated with $2 \mathrm{mM}$ of $\mathbf{3}^{\prime}$ and $40 \mu \mathrm{M}$ FAD in Tris. $\mathrm{HCl}$ buffer (pH 8.0) at $29^{\circ} \mathrm{C}$. No new product was detected after overnight incubation. Because $\mathbf{3}^{\prime}$ cannot be processed by SpnJ, it is reasonable to conclude that $\mathbf{3}$ is not a substrate for SpnJ.

In contrast, TLC and reverse phase-HPLC analysis of the incubation mixture containing SpnJ $(20 \mu \mathrm{M}), 2 \mathrm{mM}$ of $\mathbf{4}$, and $40 \mu \mathrm{M}$ FAD in Tris. $\mathrm{HCl}$ buffer ( $\mathrm{pH} 8.0$ ) revealed the time-dependent formation of a new product (nearly quantitative conversion after $4 \mathrm{~h}$ at $29^{\circ} \mathrm{C}$, see Figure 1), which was identified by ${ }^{1} \mathrm{H}$ and ${ }^{13} \mathrm{C}$ NMR spectroscopy as well as HR-CIMS analysis as the C-15 ketone derivative 5 . $^{22}$ These results clearly established that SpnJ is a flavin oxidase and 4 is the substrate for SpnJ. The fact that no trace of cross-bridging products was detected indicated that $\mathrm{SpnJ}$ is only a mono-functional oxidase. These results provide strong support for the conversion of $\mathbf{4}$ to $\mathbf{5}$ as the first step in the post-cyclization reactions (vide supra), and set the stage for the study of the putative "Diels-Alderase" activity.

In summary, the experiments described herein detail a convergent synthesis of $\mathbf{4}$ postulated to be involved in spinosyn biosynthesis. More importantly, we have validated the catalytic function of SpnJ and established $\mathbf{4}$ as the precursor of the tricyclic nucleus of spinosyns. Future work will encompass characterization of the remaining enzymes (SpnF, SpnL, and SpnM) believed to be involved in the cross-bridging event as well as their biological roles leading to an understanding of the mechanism of formation for the spinosyn aglycone.

\section{Supplementary Material}

Refer to Web version on PubMed Central for supplementary material.

\section{Acknowledgements}

This work was supported by grants from the National Institutes of Health (GM35906 and GM54346).

\section{References}

1. Kirst HA, Michel KH, Martin JW, Creemer LC, Chio EH, Yao RC, Nakatsukasa WM, Boeck LD, Occolowitz JL, Paschal JW, Deeter JB, Jones ND, Thompson GD. Tetrahedron Lett 1991;32:4839_ 4842 .

2. (a) Sparks TC, Grouse GD, Durst G. Pest Manag Sci 2001;57:896-905. [PubMed: 11695182]Salgado, VL.; Sparks, TC. Comprehensive Molecular Insect Science. Iatrou, K.; Gill, SS.; Gilbert, LI., editors. 6. Pergamon; Oxford: 2005. p. 137-173.

3. Waldron C, Matsushima P, Rosteck PR Jr, Broughton MC, Turner J, Madduri K, Crawford KP, Merlo DJ, Baltz RH. Chem Biol 2001;8:487-499. [PubMed: 11358695]

4. Martin CJ, Timoney MC, Sheridan RM, Kendrew SG, Wilkinson B, Staunton JC, Leadlay PF. Org Biomol Chem 2003;1:4144-4147. [PubMed: 14685317]

5. Gullon S, Olano C, Abdelfattah MS, Brana AF, Rohr J, Mendez C, Salas JA. Appl Environ Microbiol 2006;72:4172-4183. [PubMed: 16751529]

6. Solanapyrone Synthase: (a) Katayama K, Kobayashi T, Oikawa H, Honma M, Ichihara A. Biochim Biophys Acta 1998;1384:387-395. [PubMed: 9659400]Lavostatin biosynthesis: (b) Auclair K, Sutherland A, Kennedy J, Witter DJ, Van den Heever JP, Hutchinson CR, Vederas JC. J Am Chem Soc 2000;122:11519-11520.Macrophomate Synthase: (c) Ose T, Watanabe K, Mie T, Honma M, Watanabe H, Yao M, Oikawa H, Tanaka I. Nature 2003;422:185-189. [PubMed: 12634789] Guimaraes CR, Udier-Blagovic M, Jorgensen WL. J Am Chem Soc 2005;127:3577-3588. [PubMed: 15755179]Serafimov JM, Westfeld T, Meier BH, Hilvert D. J Am Chem Soc 2007;129:9580-9581. [PubMed: 17636922]Review: (d) Oikawa H. Bull Chem Soc Jap 2005;78:537-554.Stocking EM, Williams RM. Angew Chem Int Ed Engl 2003;42:3078-3115. [PubMed: 12866094] 
7. The configurations at C-9,11, $15^{4}$ and 17 were assigned based on the fact that the four ketoreductase (KR) domains in the spn PKS assembly are all type A KRs ( Caffrey P. Chem Bio Chem 2003;4:6546S7. ${ }^{22}$

8. Molander GA, McWilliams JC, Noll BC. J Am Chem Soc 1997;119:1265-1276.

9. Soai K, Yokoyama S, Hayasaka T, Ebihara K. Chem Lett 1988:843-846.

10. Crimmins MT, King BW, Tabet EA, Chaudhary K. J Org Chem 2001;66:894-902. [PubMed: 11430110]

11. Heathcock CH, Pirrung MC, Sohn JE. J Org Chem 1979;44:4294-4299.

12. Brown HC, Randad RS, Bhat KS, Zaidlewicz M, Racherla US. J Am Chem Soc 1990;112:2389_ 2392.

13. Rychnovsky SD, Rogers BN, Richardson TI. Acc Chem Res 1998;31:9-17.

14. Paquette LA, Chang SK. Org Lett 2005;7:3111-3114. [PubMed: 15987218]

15. Saniere M, Lemerrer Y, Barbe B, Koscielniak T, Depezay JC. Tetrahedron 1989;45:7317-28.

16. Yu W, Mei Y, Kang Y, Hua Z, Jin Z. Org Lett 2004;6:3217-9. [PubMed: 15355016]

17. Evans DA, Black WC. J Am Chem Soc 1993;115:4497-513.

18. Blakemore PR, Cole WJ, Kocienski PJ, Morley A. Synlett 1998:26-8.

19. Rodriguez J, Waegell B. Synthesis 1988:534-535.

20. Jeffery T. Tetrahedron Lett 1985;26:2667-2670.

21. Inanaga J, Hirata K, Saeki H, Katsuki T, Yamaguchi M. Bull Chem Soc Jpn 1979;52:1989-1993.

22. See Supporting Information for spectral data and experimental details. 


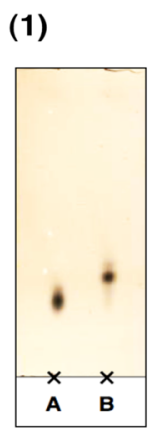

(2) 3.5

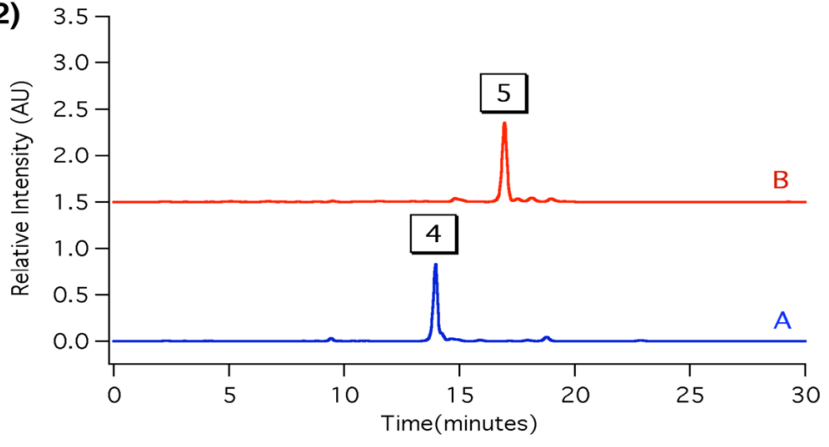

Figure 1.

(1) TLC and (2) HPLC analysis of incubation mixture without SpnJ (A) or with SpnJ (B) 21 (TLC: silical gel, $\mathrm{CH}_{2} \mathrm{Cl}_{2}: \mathrm{MeOH}=93: 7$; HPLC: $\mathrm{C}-18,2 \% \mathrm{NH}_{4} \mathrm{OAc}: \mathrm{CH}_{3} \mathrm{CN}=70: 30$ to 20:80 over $120 \mathrm{~min})$. 

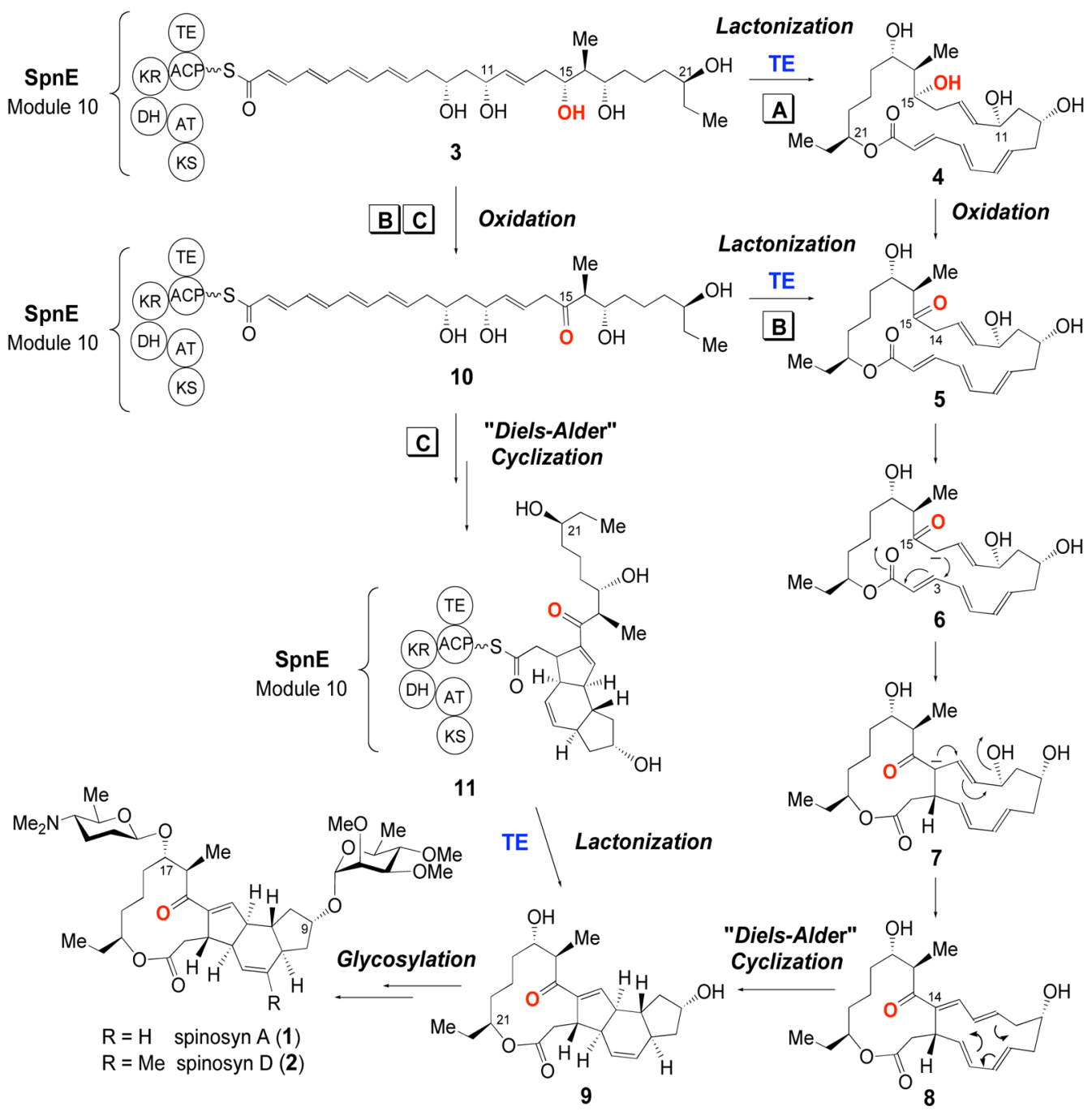

Scheme 1. 


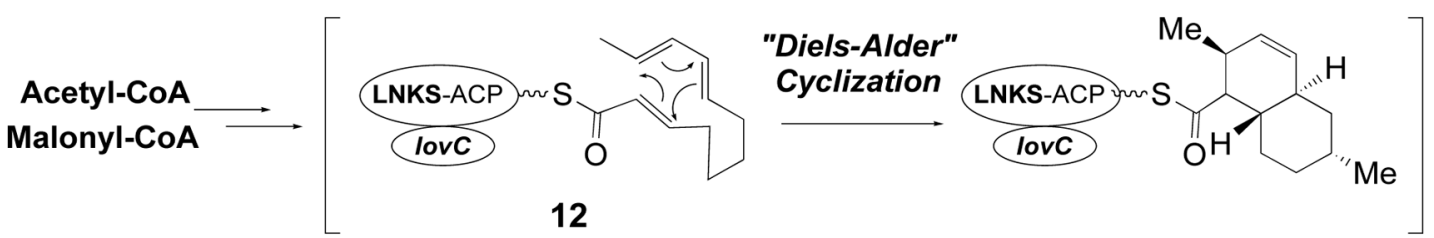

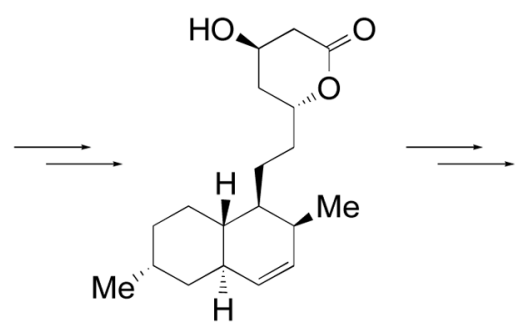

13

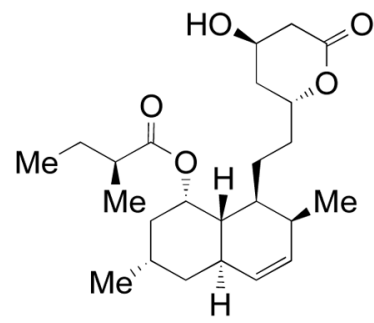

14

Scheme 2. 


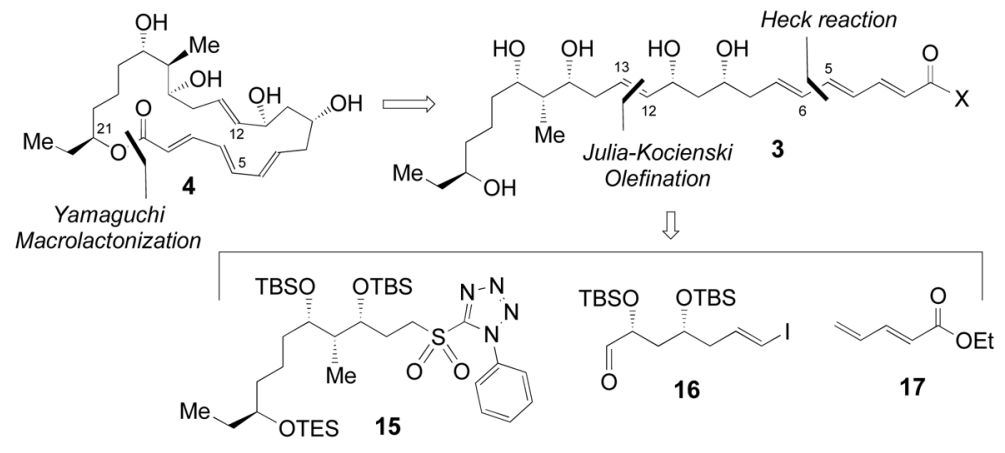

Scheme 3. 

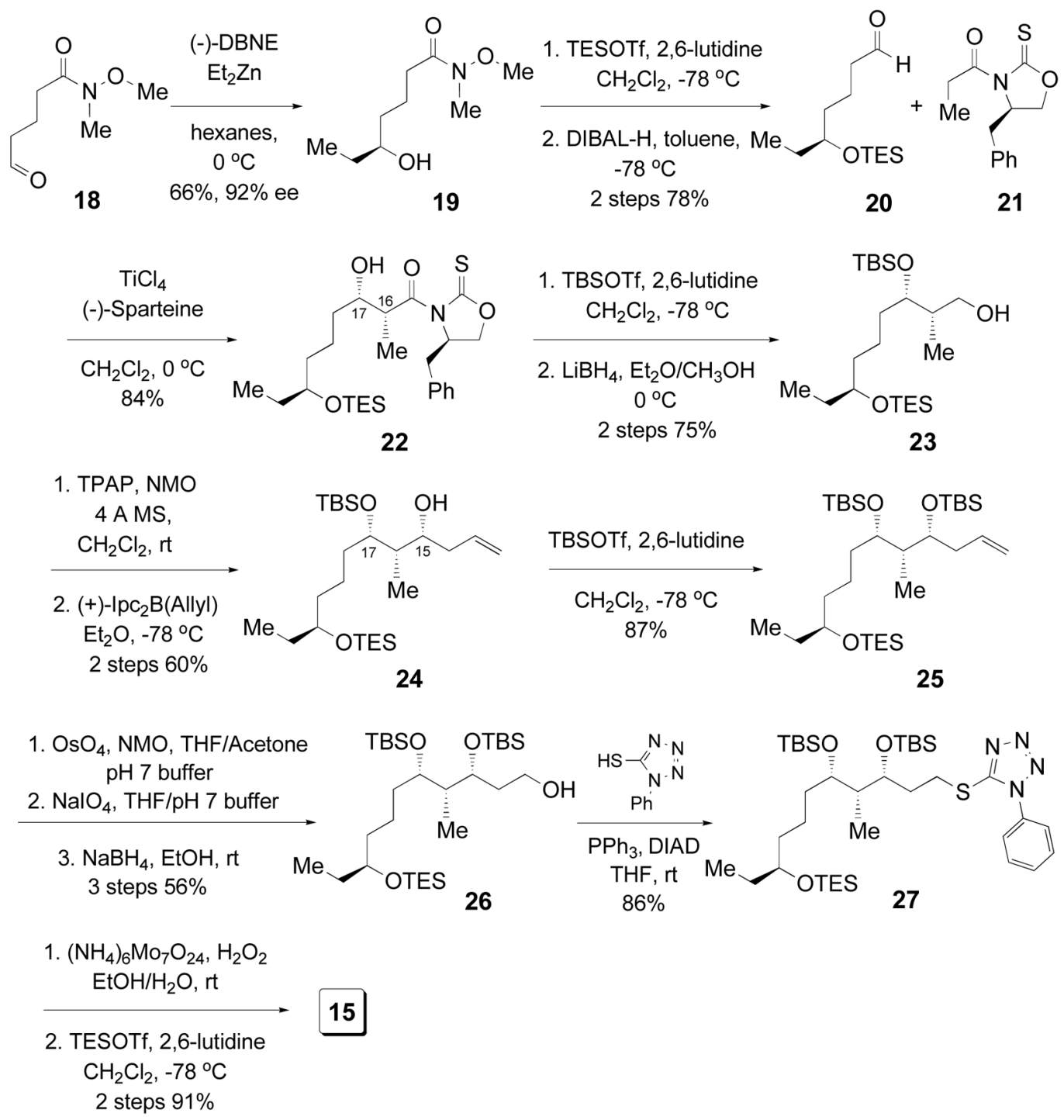

Scheme 4. 

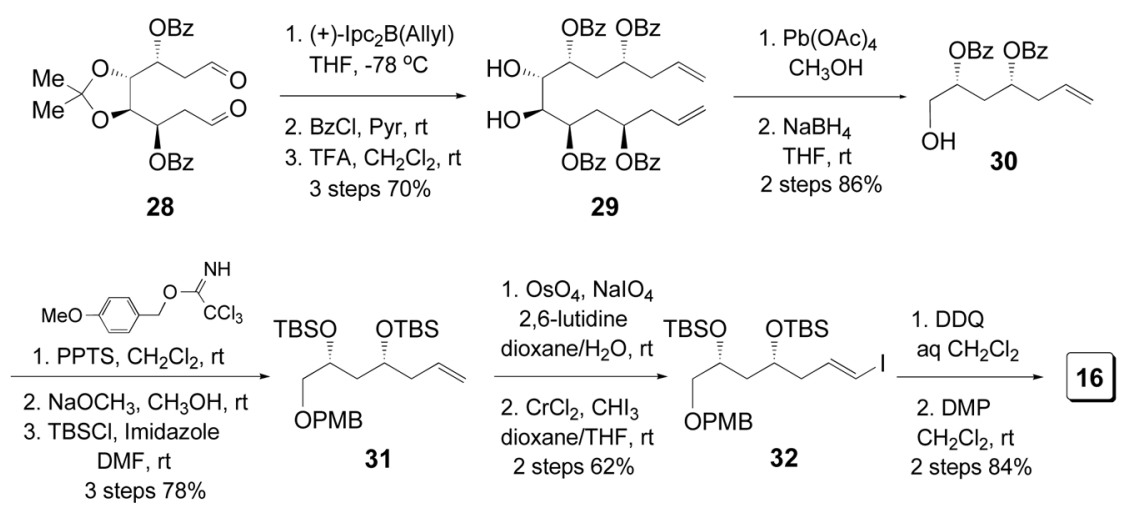

Scheme 5 . 

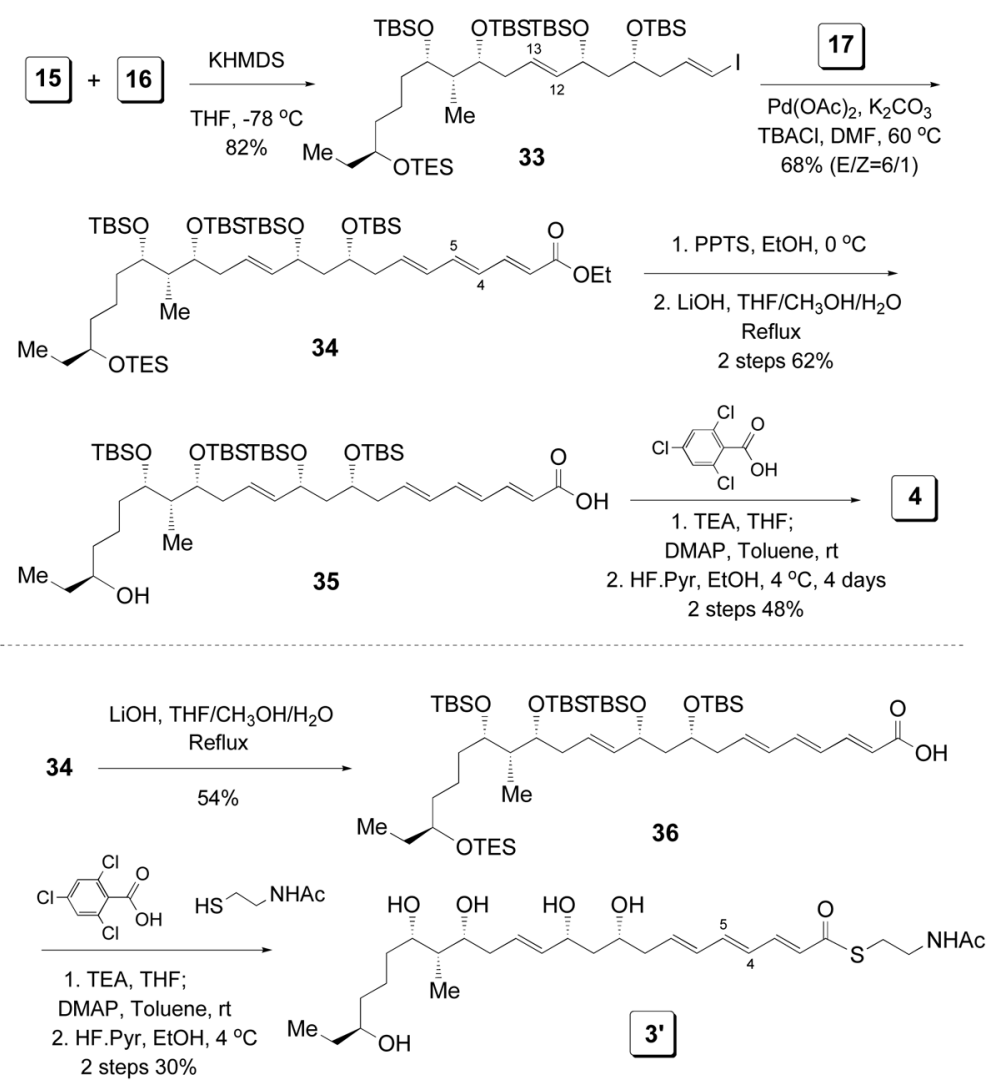

\section{Scheme 6.}

